\title{
Effect of inborn vs. outborn delivery on neurodevelopmental outcomes in infants with hypoxic-ischemic encephalopathy: secondary analyses of the NICHD whole-body cooling trial
}

\author{
Girija Natarajan', Athina Pappas', Seetha Shankaran', Abbot R. Laptook², Michele Walsh', Scott A. McDonald4, \\ Richard A. Ehrenkranz ${ }^{5}$, Jon E. Tyson ${ }^{6}$, Ronald N. Goldberg ${ }^{7}$, Rebecca Bara', Rosemary D. Higgins ${ }^{8}$, Abhik Das ${ }^{4}$ \\ and Breda Munoz ${ }^{4}$
}

\begin{abstract}
BACKGROUND: The effect of birth location on hypothermiarelated outcomes has not been rigorously examined in the literature. In this study, we determined whether birth location had an impact on the benefits of whole-body cooling to $33.5^{\circ} \mathrm{C}$ for $72 \mathrm{~h}$ in term infants $(n=208)$ with hypoxic-ischemic encephalopathy (HIE) who participated in the Neonatal Research Network (NRN) randomized controlled trial.
\end{abstract}

METHODS: Heterogeneity by birth location was examined with respect to cooling treatment for the 18-mo primary outcomes (death, moderate disability, severe disability) and secondary outcomes (death, components of disability), and in-hospital organ dysfunction. Logistic regression models were used to generate adjusted odds ratios.

RESULTS: Infants born at a location other than an NRN center (outborn) ( $n=93$ ) experienced significant delays in initiation of therapy (mean (SD): 5.5 (1.1) vs. $4.4(1.2) \mathrm{h}$ ), lower baseline temperatures (36.6 (1.2) vs. $\left.37.1(0.9){ }^{\circ} \mathrm{C}\right)$, and more severe HIE (43 vs. $29 \%)$ than infants born in an NRN center (inborn) $(n=115)$. Maternal education $<12$ y (50 vs. 14\%) and African-American ethnicity (43 vs. 25\%) were more common in the inborn group. When adjusted for NRN center and HIE severity, there were no significant differences in 18-mo outcomes or in-hospital organ dysfunction between inborn and outborn infants.

CONCLUSION: Although limited by sample size and some differences in baseline characteristics, the study showed that birth location does not appear to modify the treatment effect of hypothermia after HIE.

$\mathbf{H}$ ypoxic-ischemic encephalopathy (HIE) caused by acute perinatal asphyxia remains an important cause of neonatal mortality and neurodevelopmental deficit in childhood. The Cochrane meta-analysis of eight randomized controlled trials (RCTs) of therapeutic hypothermia in newborns with HIE demonstrated a beneficial effect for this treatment, with a statistically significant and clinically important reduction in the combined outcome of mortality and major neurodevelopmental disability up to the age of 18 mo (relative risk 0.76 ; $95 \%$ confidence interval (CI) 0.65-0.89) (1). Large published RCTs have shown a reduction in death or major developmental disability among infants with HIE who had undergone hypothermia therapy as compared with controls (Eunice Kennedy Shriver National Institute of Child Health and Human Development (NICHD) Neonatal Research Network (NRN) trial and the Infant Cooling Evaluation trial), a reduction in death or disability among infants with less severe amplitude-integrated electroencephalogram changes at randomization (CoolCap trial), and an increase in survival without disability (TOBY trial) (2-5). With the widespread adoption of therapeutic hypothermia for HIE, it has become important to ascertain factors that could modulate its effectiveness and safety.

In an early pilot study by Eicher and colleagues, infants transported into the participating centers (outborn) were found to be significantly more likely to die than those born in the participating centers (inborn), after controlling for treatment group $(6,7)$. There is scarcity of data on the effects of birth location on outcomes related to hypothermia therapy in neonatal HIE. We undertook a secondary analysis of data from the NICHD NRN whole-body hypothermia RCT to test the interaction between the hypothermia treatment and the location of birth (inborn or outborn), so as to determine whether the benefits and potential adverse effects of whole-body cooling vary in these subgroups. In addition, factors that may contribute to a difference in outcomes, if any, between the groups were examined.

\section{RESULTS}

Data on the primary outcome were available for 205 (98.5\%) of the 208 infants enrolled. The three infants for whom data were missing were in the control group. The baseline characteristics

\footnotetext{
'Department of Pediatrics, Wayne State University, Detroit, Michigan; ${ }^{2}$ Department of Pediatrics, Women and Infants Hospital, Brown University, Providence, Rhode Island; ${ }^{3}$ Department of Pediatrics, Rainbow Babies \& Children's Hospital, Case Western Reserve University, Cleveland, Ohio; ${ }^{4}$ Department of Pediatrics, Statistics and Epidemiology Unit, Research Triangle International, Research Triangle Park, North Carolina; ${ }^{5}$ Department of Pediatrics, Yale University School of Medicine, New Haven, Connecticut; ${ }^{6}$ Department of Pediatrics, University of Texas Medical School at Houston, Houston, Texas; ${ }^{7}$ Department of Pediatrics, Duke University, Durham, North Carolina; ${ }^{8}$ Department of Pediatrics, Pregnancy and Perinatology Branch, Center for Developmental Biology and Perinatal Medicine, Eunice Kennedy Shriver National Institute of Child Health and Human Development, National Institutes of Health, Bethesda, Maryland; for the Hypothermia Subcommittee of the Eunice Kennedy Shriver NICHD Neonatal Research Network. Correspondence: Girija Natarajan (gnatara@med.wayne.edu)
} 
of outborn $(n=93)$ and inborn $(n=115)$ infants are compared in Table 1. Maternal education $<12$ y (14 vs. 50\%), AfricanAmerican ethnicity (25 vs. 43\%), and maternal intrapartum fever (4 vs. 15\%) were significantly less frequent in outborn infants as compared to inborn ones. Outborn infants had (i) significantly delayed age at study entry (mean ( \pm SD): 5 (0.9) vs. $3.7(1.1) \mathrm{h}$ ); (ii) a greater proportion with time to onset of spontaneous respiration $>10 \mathrm{~min}$ (79 vs. 64\%); (iii) a greater need for continued resuscitation at $10 \mathrm{~min}$ (99 vs. $90 \%$ ); and (iv) more severe HIE (44 vs. 29\%) than inborn infants $(P<$ 0.05). Comparable proportions ( 48 vs. $53 \%$ ) in the two groups of infants were randomized to receive whole-body cooling. Outborn infants had lower baseline temperatures (36.6 (1.2) vs. $\left.37.1(0.9){ }^{\circ} \mathrm{C}\right)$ when placed on the cooling blanket, a greater delay in the initiation of cooling (mean ( \pm SD): 5.5 (1.1) vs. 4.4 $(1.2) \mathrm{h}$ ), and higher age at target temperature (mean $( \pm \mathrm{SD})$ : $6.4(1.2)$ vs. $5.6(1.3) \mathrm{h}$ ) than inborn infants.

Figure 1 reflects the outcomes related to hypothermia in outborn and inborn infants. The rates of adverse outcomes, namely, (i) death or moderate or severe disability, (ii) death, (iii) Mental Developmental Index (MDI) < 70, and (iv) Psychomotor Developmental Index $<70$, were all higher in the outborn population than in the inborn population, in both hypothermiatreated and control subgroups. Table 2 shows the odds ratios (OR) with corresponding 95\% CI and $P$ values for interaction for each outcome in outborn and inborn subgroups after adjustment for study center and for the severity of HIE. There was no significant heterogeneity of treatment effect for any outcome assessed as shown by unadjusted analysis, by analysis after adjustment for study center, or by analysis after adjustment for both study center and severity of HIE.

\section{DISCUSSION}

Therapeutic hypothermia for neonatal HIE has been demonstrated to have a consistent benefit without major adverse effects $(1,8-10)$. We utilized data from the NICHD NRN whole-body cooling RCT to determine whether the safety and efficacy of hypothermia varied between a group of infants who were inborn and a group of infants transported from a referral center to an NRN site. The unadjusted rates of adverse outcomes (death or moderate or severe disability; death; MDI $<70$; and psychomotor developmental index $<70$ ) were higher in the outborn population as compared with the inborn population in both hypothermia-treated and control subgroups. After adjusting for NRN center and severity of encephalopathy, we were unable to detect an independent effect of birth location on outcome.

In an early pilot study by Eicher and colleagues, outborn infants, who formed $75 \%$ of the study population, were found to be significantly more likely to die than inborn infants (OR 10.7; 95\% CI 1.3-90), after controlling for treatment group. However, severity of HIE was not controlled for in this analysis (7). Approximately $77 \%$ of the study cohort had severe HIE (Sarnat stage III). Of 24 deaths, 22 occurred in the outborn population. Active cooling was undertaken during transport for all outborn infants. Of note, in the infants who received
Table 1. Baseline characteristics of outborn and inborn groups of infants

\begin{tabular}{|c|c|c|c|}
\hline Characteristic, mean (SD) or \% & $\begin{array}{c}\text { Outborn } \\
(n=93)\end{array}$ & $\begin{array}{l}\text { Inborn } \\
(n=115)\end{array}$ & $P$ value \\
\hline Maternal age (y) & $27.9(5.7)$ & $26.8(6.3)$ & 0.17 \\
\hline Maternal education < 12 y (\%) & 14.1 & 50 & $<0.0001^{*}$ \\
\hline \multicolumn{4}{|l|}{ Ethnicity (\%) } \\
\hline Black & 24.7 & 42.6 & $0.007^{*}$ \\
\hline Caucasian & 54.8 & 18.3 & $<0.0001^{*}$ \\
\hline Other & 20.4 & 39.1 & $0.006^{*}$ \\
\hline Prenatal care (\%) & 94.6 & 93.9 & 1.0 \\
\hline \multicolumn{4}{|l|}{ Pregnancy complications (\%) } \\
\hline Antepartum hemorrhage & 18.3 & 11.3 & 0.19 \\
\hline Chronic hypertension & 8.6 & 15.6 & 0.22 \\
\hline Diabetes & 4.3 & 11.3 & 0.11 \\
\hline \multicolumn{4}{|l|}{ Intrapartum complications (\%) } \\
\hline Fetal decelerations & 72.8 & 74.8 & 0.87 \\
\hline Cord prolapse & 23.7 & 13.0 & 0.07 \\
\hline Uterine rupture & 11.8 & 15.6 & 0.55 \\
\hline Shoulder dystocia & 7.6 & 11.3 & 0.51 \\
\hline Maternal fever (\%) & 4.3 & 14.8 & $0.02 *$ \\
\hline $\begin{array}{l}\text { Maternal antibiotics for suspected/ } \\
\text { confirmed infection (\%) }\end{array}$ & 16.3 & 20.2 & 0.59 \\
\hline Rupture of membranes > $18 \mathrm{~h}(\%)$ & 8.6 & 9.2 & 1.00 \\
\hline Maternal hemorrhage (\%) & 3.2 & 9.6 & 0.12 \\
\hline Emergency cesarean delivery (\%) & 77.4 & 69.6 & 0.27 \\
\hline Birth weight (grams) & $3,422(594)$ & $3,341(658)$ & 0.36 \\
\hline Gestational age (wk) & $38.8(1.5)$ & $38.9(1.7)$ & 0.62 \\
\hline Male gender (\%) & 57 & 56 & 0.96 \\
\hline \multicolumn{4}{|l|}{ Apgar score < 5 (\%) } \\
\hline At $5 \mathrm{~min}$ & 88.0 & 79.1 & 0.13 \\
\hline At $10 \mathrm{~min}$ & 75.9 & 65.4 & 0.16 \\
\hline Age at admission (h) & $5.0(0.9)$ & $3.7(1.1)$ & $<0.0001^{*}$ \\
\hline Age therapy started (h) & $5.5(1.0)$ & $4.3(1.2)$ & $<0.0001^{*}$ \\
\hline Age at target temperature $(\mathrm{h})$ & $6.4(1.2)$ & $5.6(1.3)$ & $0.002^{*}$ \\
\hline Temperature at randomization $\left({ }^{\circ} \mathrm{C}\right)$ & $36.6(1.2)$ & $37.1(0.9)$ & $0.002^{*}$ \\
\hline $\begin{array}{l}\text { Temperature }>37.5^{\circ} \mathrm{C} \text { at } \\
\text { randomization (\%) }\end{array}$ & 17.0 & 26.6 & 0.15 \\
\hline DR intubation (\%) & 94.6 & 93.0 & 0.86 \\
\hline $\begin{array}{l}\text { Continued resuscitation at } \\
10 \mathrm{~min}(\%)\end{array}$ & 98.9 & 89.6 & $0.01 *$ \\
\hline $\begin{array}{l}\text { Time of spontaneous } \\
\text { respiration }>10 \min (\%)\end{array}$ & 79.5 & 64.2 & $0.03^{*}$ \\
\hline Cord pH & $6.82(0.19)$ & $6.88(0.21)$ & 0.11 \\
\hline Base deficit & $19.9(7.6)$ & $18.9(7.8)$ & 0.49 \\
\hline Severity of encephalopathy (\%) & & & $0.04 *$ \\
\hline Moderate & 56.5 & 71.3 & \\
\hline Severe & 43.5 & 28.7 & \\
\hline Cooling group (\%) & 48.4 & 53 & 0.60 \\
\hline $\begin{array}{l}\text { Septicemia/meningitis/ } \\
\text { encephalitis (\%) }\end{array}$ & 5.4 & 5.2 & 1.0 \\
\hline
\end{tabular}

DR, delivery room.

*Significant difference at 5\% level. 


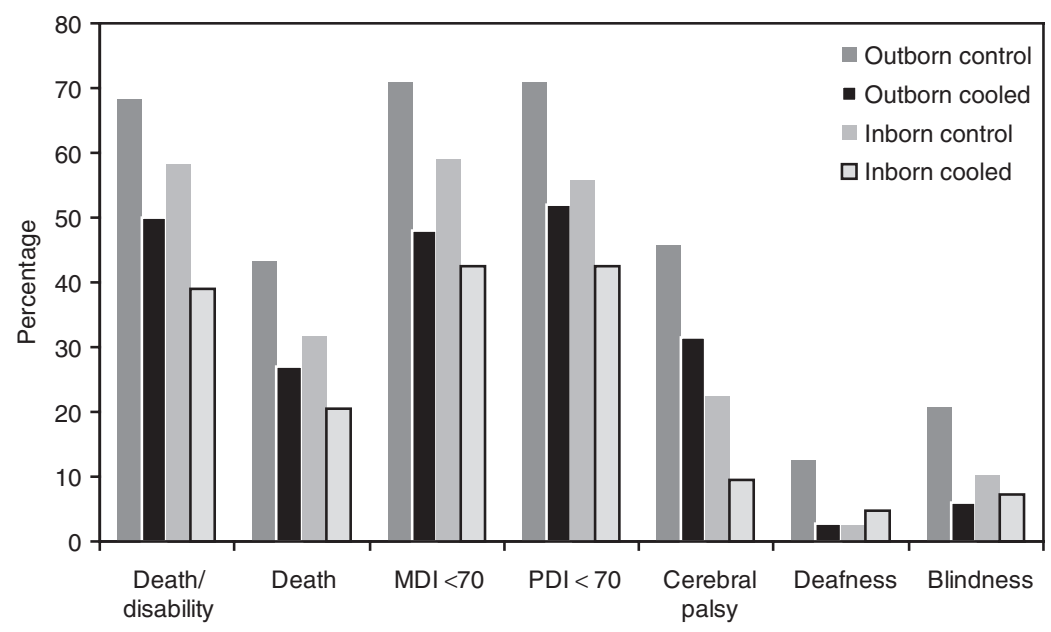

Figure 1. Rates of 18- to 22-mo outcomes related to hypothermia treatment in outborn and inborn groups of infants. The dark gray bar represents outborn control infants, the black bar represents outborn hypothermia-treated infants, the medium-gray bar represents inborn controls, and the light-gray outlined bar represents the inborn hypothermia-treated infants. MDI, Mental Developmental Index; PDI, Psychomotor Developmental Index.

hypothermia treatment, the mean time to target temperature $\left(32.5-33.5^{\circ} \mathrm{C}\right)$ in outborn infants $(n=23)$ tended to be shorter than in inborn ones $(n=6)(111 \pm 78$ vs. $156 \pm 116 \mathrm{~min})$. The authors speculated that the adverse outcomes among outborn infants may have been due to lack of experienced personnel, difficulties in resuscitation, or problems with postresuscitation stabilization. In the recent ICE trial there was no significant finding to suggest that the effects of hypothermia differed within the outborn subgroup (5). The ICE trial also included cooling at the birth hospital and passive and active cooling during transport, along with rectal temperature monitoring (5). However, in the current NICHD NRN data set, cooling was not undertaken for the infants during transport.

In this study, a significantly greater proportion of outborn infants (as compared to inborn ones) required continued resuscitation at $10 \mathrm{~min}$, experienced onset of spontaneous respiration at $>10 \mathrm{~min}$, and had severe, rather than moderate HIE. It was not possible to determine whether these differences originated from any systematic differences in the availability of personnel or quality and effectiveness of resuscitation, or whether it reflected a referral bias, with the most severely HIEaffected infants being referred to another center for cooling.

We noted several differences in baseline characteristics between the inborn and outborn groups. Maternal fever occurred more often, and baseline infant temperatures were higher in the inborn group. Elevated temperatures $>37.5^{\circ} \mathrm{C}$ were noted in $27 \%$ of inborn infants as compared to $17 \%$ of the outborn ones; however, these differences were not statistically significant. Elevated temperatures during study intervention among control infants have been previously shown to increase the odds for death or disability $(11,12)$. On the other hand, outborn infants experienced a greater delay in initiation of cooling therapy (although still within the 6-h window of the protocol), and took longer to attain target temperatures. Experimental data in animal models suggest that cerebral hypothermia that is initiated as early as possible in the latent phase of injury is associated with neuroprotection (13). In the CoolCap trial, within the fairly narrow range of recruitment times, there was no greater improvement in those treated earliest after birth than in those treated later (4). In the NICHD study cohort, time to achieve target temperatures did not influence outcome (8). In the TOBY trial, the effect of treatment group did not vary significantly on the basis of time to randomization: among the 105 infants randomly assigned to a group at $<4 \mathrm{~h}$ after birth, the relative risk for the primary outcome (death or severe neurodevelopmental disability) with hypothermia treatment was 0.77 (95\% CI 0.44-1.04), whereas among the other 220 infants who were randomly assigned to groups at $4-6 \mathrm{~h}$ after birth, the relative risk was 0.95 (95\% CI $0.72-1.25 ; P=0.21$ for interaction) (3). In the ICE trial, no significant interactions were demonstrated between hypothermia and age at randomization $(P=0.22)(5)$. The age at randomization in the CoolCap, NICHD, TOBY, and ICE RCTs were 4.8 (range 2.6-6), mean 4.3 (SD 1.3), median 4.7 (range 3.8-5.4), and mean (SD) 4 (1.3) $\mathrm{h}$, respectively. In recent years, with the premise that "time to target temperature" may be critical, there have been reports of cooling of the infants being carried out during transport to the treatment center (14-16). Passive, active, and servo-controlled cooling during transport have been performed in small numbers of infants (14-16). However, overcooling to $<32^{\circ} \mathrm{C}$ has been reported in one-third of the infants; therefore it is critical to monitor core temperatures when carrying out cooling in infants during transport. More research is needed on safety and efficacy issues of cooling treatment during transport. Early ascertainment of encephalopathy is also essential so as to optimally disseminate therapeutic hypothermia to centers that do not currently offer this therapy $(14,15)$.

The reason for more elevated temperatures recorded in inborn infants is unclear, although they could be secondary to higher maternal temperatures. Maternal antibiotic regimens, conditions of prolonged rupture of membranes, and clinical diagnosis of neonatal septicemia, all of which are used as surrogate measures of infection, were not different between the groups. The primary trial was completed under conditions of 
Table 2. ORs with corresponding $95 \% \mathrm{Cls}$ and $P$ values for interaction for each outcome in outborn and inborn subgroups after adjustment for center and severity of encephalopathy

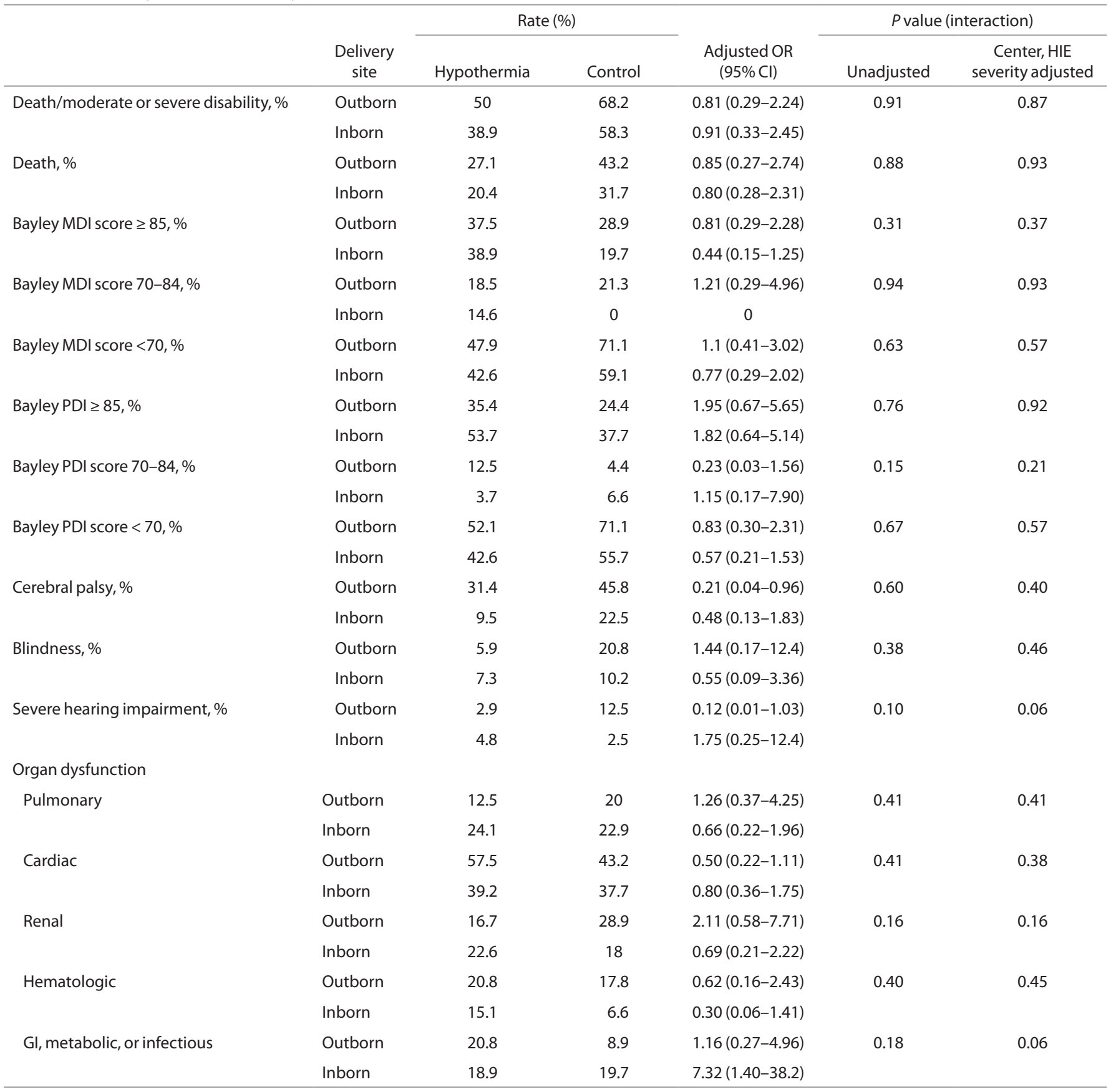

Cl, confidence interval; Gl, gastrointestinal; HIE, hypoxic-ischemic encephalopathy; MDI, Mental Developmental Index; OR, odds ratio; PDI, Psychomotor Developmental Index.

clinical equipoise, and cooling was not initiated prior to the trial intervention at the NRN center. It is unlikely, therefore, that the differences in temperatures were related to transport conditions. Another significant difference between the groups was in the frequency of low maternal education $(<12$ y) $(50 \%$ in the inborn population vs. $14 \%$ among the outborn), a potential modulator of early childhood developmental outcomes.

The strengths of our study are that data were collected prospectively from infants diagnosed with HIE using stringent clinical and biochemical criteria and managed according to a consistent protocol in tertiary level centers. We had a very high follow-up rate, and a structured neurodevelopmental assessment was performed at $18-22$ mo by certified trained examiners. The proportion of outborn vs. inborn infants in the trial was roughly equal (45 vs. $55 \%$ ), allowing a reasonable statistical comparison. The primary trial was completed at a time when there was clinical equipoise and cooling was not initiated prior to the trial intervention at the NRN center.

The reasons for the absence of any significant interaction between birth location and the effects of cooling are complex. 
Baseline differences between groups, specifically the higher baseline temperatures and lower maternal education levels in inborn infants as a group, may have obscured an effect on outcomes. We may have had relatively low power to detect an interaction in this secondary analysis. Nonetheless, the value of a careful statistical exploration for heterogeneity of treatment effect, based on clinical data and biologic rationale, has been previously recognized (17). Gabler and colleagues recently found that only $29 \%$ of the RCTs that reported results had formally analyzed "heterogeneity of treatment effects" data (18). They suggest that heterogeneity of treatment effect may have important implications for individualized patient care, and that therefore the lack of such analyses represent "missed opportunities" (18).

We examined the putative association between outcomes and birth location, potentially important data for risk stratification and optimizing outcomes in perinatal HIE. In conclusion, although limited by sample size and some differences in baseline characteristics, birth location does not appear to modify the treatment effect of hypothermia treatment after HIE in newborn infants.

\section{METHODS}

This was a secondary post hoc analysis of data collected as part of the whole-body cooling NICHD NRN RCT (2). The safety and 18-mo neurodevelopmental outcomes of the trial have been previously reported $(2,8)$. The original trial was approved by the institutional review board at each participating site, and written consent was obtained from the parents of eligible infants.

The study population was composed of infants with HIE, enrolled in the whole-body cooling NICHD NRN RCT (2). Eligibility criteria included $\mathrm{pH}$ level of $\leq 7$ or a base deficit of $\geq 16$ in cord blood or during the first hour after birth. If $\mathrm{pH}$ was between 7.01 and 7.15 during this interval and if base deficit was between 10 and 15.9 (or if blood gas data were not available), the additional criteria required included an acute perinatal event (late or variable decelerations, cord prolapse, cord rupture, uterine rupture, maternal trauma, hemorrhage, or cardio-respiratory arrest), and either a 10-min Apgar score of $\leq 5$ or assisted ventilation initiated at birth and continued for at least $10 \mathrm{~min}$ (2). All the infants were moderately or severely encephalopathic (one or more signs in at least three of six categories: level of consciousness, spontaneous activity, posture, tone, primitive reflexes, and autonomic nervous system) on a standardized neurologic examination by a certified physician examiner, or had seizures. Exclusion criteria were inability to enroll the infant before $6 \mathrm{~h}$ of life, major congenital anomaly, birth weight $<1800$ grams, refusal of consent by parent or attending physician, and moribund infant for whom no further aggressive treatment was planned. The infants were randomly assigned to treatment groups stratified by center. The neurodevelopmental assessments at 18-22 mo of age were performed by trained examiners who were unaware of the intervention status of the infants.

"Inborn" was defined in the original whole-body cooling trial as birth at a site performing the trial. "Outborn" included infants transferred to the clinical site where the hypothermia trial was conducted; the birth could have been in a hospital setting or outside of a hospital or healthcare setting such as delivery at home or in the ambulance. The interaction between cooling and inborn vs. outborn birth was examined using 18-mo primary outcomes (death or moderate or severe disability) and secondary outcomes (components of the primary outcome separately) of the primary trial, and organ dysfunction (safety outcome) during hospitalization. Severe disability was defined as (i) MDI $<70$ on Bayley Scales of Infant Development II, (ii) gross motor functional classification scale (Palisano) level 3-5, (iii) hearing impairment requiring aids ( $>60 \mathrm{db}$ audiometric testing), or (iv) bilateral blindness ( $<20 / 200$ visual acuity). Moderate disability was defined as MDI of 70 to 84 plus one of the following: (i) gross motor functional classification scale level 2 , (ii) hearing impairment requiring no amplification, or (iii) a persistent seizure disorder defined as recurrent seizures after Neonatal Intensive Care Unit discharge, requiring anti-convulsant therapy at the 18-mo examination time point. Non-central nervous system organ dysfunction was defined as follows: (i) pulmonary involvement included meconium aspiration syndrome, persistent pulmonary hypertension of the newborn (requiring inhaled nitric oxide and extra-corporeal membrane oxygenation), chronic lung disease, and/or pulmonary hemorrhage; (ii) cardiac involvement such as cardiomegaly, cardiac failure and cardiac dysfunction as determined by echocardiography, cardiac ischemia as determined by electrocardiography or elevated enzyme levels, hypotension, hypertension, and/or arrhythmia; (iii) renal involvement such as oliguria, anuria, and/or need for dialysis; (iv) gastrointestinal involvement characterized by hepatic dysfunction or necrotizing enterocolitis; (v) infections such as bloodstream infection, meningitis, or encephalitis; (vi) hematologic dysfunction, including thrombocytopenia and disseminated intravascular coagulation, and (vii) metabolic dysfunction, including hypoglycemia, hypocalcemia, and/or hypomagnesemia (8). Demographic data and baseline clinical characteristics of inborn and outborn groups were compared.

\section{Statistical Analysis}

Descriptive statistics were expressed as median (range), mean (SD), and number (percentage) as appropriate. Formal statistical comparisons of mean values or proportions between the two groups were carried out using two-sample $t$-test and $\chi^{2}$, as appropriate. For each dichotomous outcome, logistic regression models were fitted for the inborn and outborn subgroups, to determine an OR and 95\% CI for each subgroup. ORs were adjusted to control for NRN center and severity of HIE, two potential confounding variables that had been adjusted for in the original trial and were selected $a$ priori. The statistical significance of the heterogeneity of treatment effect was determined using logistic regression models with treatment/subgroup interactions. A value of $P<0.05$ for the test of heterogeneity (treatment X subgroup interaction) was considered a significant subgroup effect.

\section{STATEMENT OF FINANCIAL SUPPORT}

The National Institutes of Health and the Eunice Kennedy Shriver National Institute of Child Health and Human Development (NICHD) provided grant support for the Neonatal Research Network's Whole-Body Hypothermia Trial. Grant funding was provided by Alpert Medical School of Brown University and Women \& Infants Hospital of Rhode Island (U10 HD27904); Case Western Reserve University, Rainbow Babies \& Children's Hospital (U10 HD21364, M01 RR80); Cincinnati Children's Hospital Medical Center, University Hospital and Good Samaritan Hospital (U10 HD27853, M01 RR8084); Duke University, School of Medicine University Hospital, Alamance Regional Medical Center, and Durham Regional Hospital (U10 HD40492, M01 RR30); Emory University, Children's Healthcare of Atlanta, Grady Memorial Hospital, and Emory University Hospital Midtown (U10 HD27851, M01 RR39); Indiana University, University Hospital, Methodist Hospital, Riley Hospital for Children, and Wishard Health Services (U10 HD27856, M01 RR750); RTI International (U10 HD36790); Stanford University and Lucile Packard Children's Hospital (U10 HD27880, M01 RR70); University of Alabama at Birmingham Health System and Children's Hospital of Alabama (U10 HD34216, M01 RR32); University of California-San Diego Medical Center and Sharp Mary Birch Hospital for Women and Newborns (U10 HD40461); University of Miami Holtz Children's Hospital (U10 HD21397, M01 RR16587); University of Rochester Medical Center Golisano Children's Hospital (U10 HD40521, M01 RR44); University of Texas Southwestern, Medical Center at Dallas Parkland Health \& Hospital System and Children's Medical Center Dallas (U10 HD40689, M01 RR633); University of Texas Health Science Center at Houston, Medical School, Children's Memorial Hermann Hospital, and Lyndon Baines Johnson General Hospital/Harris County Hospital District (U10 HD21373); Wayne State University, Hutzel Women's Hospital and Children's Hospital of Michigan (U10 HD21385); and Yale University, Yale-New Haven Children's Hospital (U10 HD27871, UL1 RR24139, GCRC MO1 RR125, M01 RR6022).

\section{ACKNOWLEDGMENTS}

We are indebted to our medical and nursing colleagues and the infants and their parents who agreed to take part in this study. The following investigators, in addition to those listed as authors, participated in this study: NRN 
Steering Committee Chairs: Alan H. Jobe, University of Cincinnati; Michael S. Caplan, University of Chicago, Pritzker School of Medicine. Alpert Medical School of Brown University and Women \& Infants Hospital of Rhode Island: William Oh, Betty R. Vohr, Angelita M. Hensman, and Lucy Noel. Case Western Reserve University, Rainbow Babies \& Children's Hospital: Avroy A. Fanaroff, Deanne E. Wilson-Costello, Nancy S. Newman, and Bonnie S. Siner. Cincinnati Children's Hospital Medical Center, University Hospital and Good Samaritan Hospital: Kurt Schibler, Edward F. Donovan, Jean J. Steichen, Barbara Alexander, Cathy Grisby, Marcia Worley Mersmann, Holly L. Mincey, Jody Hessling, and Teresa L. Gratton. Duke University, School of Medicine University Hospital, Alamance Regional Medical Center, and Durham Regional Hospital: C. Michael Cotten, Ricki F. Goldstein, Kathy J. Auten, and Melody B. Lohmeyer. Emory University, Children's Healthcare of Atlanta, Grady Memorial Hospital, and Emory University Hospital Midtown: Barbara J. Stoll, Lucky Jain, Ira Adams-Chapman, and Ellen C. Hale. Eunice Kennedy Shriver National Institute of Child Health and Human Development: Linda L. Wright and Elizabeth M. McClure. Indiana University, University Hospital, Methodist Hospital, Riley Hospital for Children, and Wishard Health Services: Brenda B. Poindexter, James A. Lemons, Anna M. Dusick, Lucy C. Miller, Leslie Richard. RTI International: W. Kenneth Poole, Dennis Wallace, Jane Hammond, Betty K. Hastings, Jamie E. Newman, Carolyn M. Petrie Huitema, and Jeanette O'Donnell Auman. Stanford University and Lucile Packard Children's Hospital: Krisa P. Van Meurs, David K. Stevenson, Susan R. Hintz, William D. Rhine, M. Bethany Ball, Anne M. DeBattista, Barry E. Fleisher, Jean G. Kohn, Joan M. Baran, and Julie C. Lee-Ancajas. University of Alabama at Birmingham Health System and Children's Hospital of Alabama: Waldemar A. Carlo, Myriam Peralta-Carcelen, Monica V. Collins, Shirley S. Cosby, and Vivien A. Phillips. University of California - San Diego Medical Center and Sharp Mary Birch Hospital for Women and Newborns: Neil N. Finer, Yvonne E. Vaucher, Maynard R. Rasmussen, David Kaegi, Kathy Arnell, Clarence Demetrio, Martha G. Fuller, Chris Henderson, and Wade Rich. University of Miami Holtz Children's Hospital: Shahnaz Duara, Charles R. Bauer, Ruth Everett-Thomas, and Amy Mur Worth. University of Rochester Medical Center Golisano Children's Hospital: Ronnie Guillet, Dale L. Phelps, Gary J. Myers, Linda J. Reubens, Diane Hust, Julie Babish Johnson, Rosemary L. Jensen, Emily Kushner, Joan Merzbach, and Kelley Yost. University of Texas Southwestern, Medical Center at Dallas Parkland Health \& Hospital System and Children's Medical Center Dallas: Abbot R. Laptook, Pablo J. Sánchez, Walid A. Salhab, R. Sue Broyles, Roy J. Heyne, Susie Madison, Jackie F. Hickman, Gaynelle Hensley, Nancy A. Miller, Alicia Guzman, Janet S. Morgan, Cristin Dooley, Catherine Twell Boatman, Elizabeth Heyne, Linda A. Madden, and Sally S. Adams. University of Texas Health Science Center at Houston, Medical School, Children's Memorial Hermann Hospital, and Lyndon Baines Johnson General Hospital/Harris County Hospital District: Kathleen A. Kennedy, Esther G. Akpa, Patty A. Cluff, Claudia I. Franco, Anna E. Lis, Georgia E. McDavid, Maegan C. Simmons, Patti L. Pierce Tate, Nora I. Alaniz, Pamela J. Bradt, Magda Cedillo, Susan Dieterich, Terri Major-Kincade, Brenda H. Morris, and Laura L. Whitely. Wayne State University, Hutzel Women's Hospital and Children's Hospital of Michigan: Yvette R. Johnson, Geraldine Muran, Deborah Kennedy, and Laura A. Goldston.Yale University, Yale-New Haven Children's Hospital: Patricia Gettner, Monica Konstantino, JoAnn Poulsen, Elaine Romano, and Joanne Williams. All authors have made substantive intellectual contributions to the study conception and design, acquisition of data, or analysis and interpretation of data. They have all contributed to the drafting of the article or to the critical revision of it for important intellectual content, and have approved the final version to be published. Each author has participated sufficiently in the work to take public responsibility for appropriate portions of the content. Data collected at participating sites of the NICHD Neonatal Research Network (NRN) were transmitted to RTI International, the data coordinating center (DCC) for the network, which stored, managed, and analyzed the data for this study. On behalf of the NRN, Abhik Das (DCC principal investigator) and Carla Bann (DCC statistician) had full access to all the data in the study and take responsibility for the integrity of the data and accuracy of the data analysis.

\section{REFERENCES}

1. Jacobs S, Hunt R, Tarnow-Mordi W, Inder T, Davis P. Cooling for newborns with hypoxic ischaemic encephalopathy. Cochrane Database Syst Rev 2007; 4:CD003311.

2. Shankaran S, Laptook AR, Ehrenkranz RA, et al. Whole-body hypothermia for neonates with hypoxic-ischemic encephalopathy. $\mathrm{N}$ Engl J Med 2005;353:1574-84.

3. Azzopardi DV, Strohm B, Edwards AD, et al. Moderate hypothermia to treat perinatal asphyxial encephalopathy. N Engl J Med 2009;361:1349-58.

4. Gluckman PD, Wyatt JS, Azzopardi D, et al. Selective head cooling with mild systemic hypothermia after neonatal encephalopathy: multicentre randomised trial. Lancet 2005;365:663-70.

5. Jacobs SE, Morley CJ, Inder TE, et al. Whole-body hypothermia for term and near-term newborns with hypoxic-ischemic encephalopathy: a randomized controlled trial. Arch Pediatr Adolesc Med 2011;165:692-700.

6. Eicher DJ, Wagner CL, Katikaneni LP, et al. Moderate hypothermia in neonatal encephalopathy: efficacy outcomes. Pediatr Neurol 2005;32:11-7.

7. Eicher DJ, Wagner CL, Katikaneni LP, et al. Moderate hypothermia in neonatal encephalopathy: safety outcomes. Pediatr Neurol 2005;32:18-24.

8. Shankaran S, Pappas A, Laptook AR, et al. Outcomes of safety and effectiveness in a multicenter randomized, controlled trial of whole-body hypothermia for neonatal hypoxic-ischemic encephalopathy. Pediatrics 2008;122:e791-8.

9. Azzopardi D, Strohm B, Edwards AD, et al. Treatment of asphyxiated newborns with moderate hypothermia in routine clinical practice: how cooling is managed in the UK outside a clinical trial. Arch Dis Child Fetal Neonatal Ed 2009;94:F260-4.

10. Edwards AD, Brocklehurst P, Gunn AJ, et al. Neurological outcomes at 18 months of age after moderate hypothermia for perinatal hypoxic ischaemic encephalopathy: synthesis and meta-analysis of trial data. BMJ 2010;340:c363.

11. Laptook A, Tyson J, Shankaran S, et al. Elevated temperature after hypoxic-ischemic encephalopathy: risk factor for adverse outcomes. Pediatrics 2008;122:491-9.

12. Wyatt JS, Gluckman PD, Liu PY, et al. Determinants of outcomes after head cooling for neonatal encephalopathy. Pediatrics 2007;119:912-21.

13. Gunn AJ. Cerebral hypothermia for prevention of brain injury following perinatal asphyxia. Curr Opin Pediatr 2000;12:111-5.

14. Fairchild K, Sokora D, Scott J, Zanelli S. Therapeutic hypothermia on neonatal transport: 4-year experience in a single NICU. J Perinatol 2010;30:324-9.

15. Hallberg B, Olson L, Bartocci M, Edqvist I, Blennow M. Passive induction of hypothermia during transport of asphyxiated infants: a risk of excessive cooling. Acta Paediatr 2009;98:942-6.

16. Johnston ED, Becher JC, Mitchell AP, Stenson BJ. Provision of servocontrolled cooling during neonatal transport. Arch Dis Child Fetal Neonatal Ed 2011; e-pub ahead of print 6 March 2011.

17. Kent DM, Rothwell PM, Ioannidis JP, Altman DG, Hayward RA. Assessing and reporting heterogeneity in treatment effects in clinical trials: a proposal. Trials 2010;11:85.

18. Gabler NB, Duan N, Liao D, Elmore JG, Ganiats TG, Kravitz RL. Dealing with heterogeneity of treatment effects: is the literature up to the challenge? Trials 2009;10:43. 